\title{
Age estimation by pulp/tooth ratio in lateral and central incisors by peri-apical $\mathrm{X}$-ray
}

R. Cameriere PhD Forensic Odontologist ${ }^{\text {a }}$, E. Cunha PhD Professor ${ }^{\text {b, }}$ S.N. Wasterlain PhD

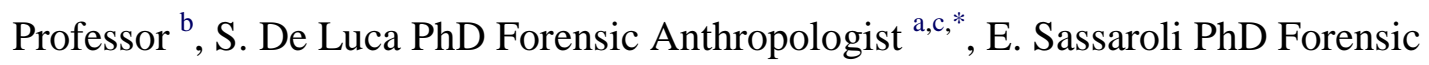
Odontologist ${ }^{\mathrm{d}}$, F. Pagliara PhD Forensic Odontologist ${ }^{\mathrm{d}}$, E. Nuzzolese PhD Forensic Odontologist $^{\mathrm{e}}$, M. Cingolani PhD Professor ${ }^{\mathrm{f}}$, L. Ferrante PhD Professor ${ }^{\mathrm{g}}$

${ }^{a}$ AgEstimation Project, Institute of Legal Medicine, University of Macerata, Macerata,

Italy

${ }^{\text {b } C e n t r o ~ d e ~ I n v e s t i g a c ̧ a ̃ o ~ e m ~ A n t r o p o l o g i a ~ e ~ S a u ́ d e, ~ D e p a r t m e n t ~ o f ~ L i f e ~ S c i e n c e s, ~}$ University of Coimbra, Coimbra, Portugal

${ }^{\mathrm{c}}$ Calle Gran Canaria, 19, 2o 1a, 08205 Sabadell, Barcelona, Spain

${ }^{\mathrm{d}}$ AgEstimation Project, Italy

e AgEstimation Project, Bari, Italy

${ }^{\mathrm{f}}$ Institute of Legal Medicine, University of Macerata, Italy

${ }^{\mathrm{g}}$ Department of Biomedical Sciences and Public Health, Faculty of Medicine, Polytechnic University of Marche, Ancona, Italy

Keywords:

Forensic sciences

Age estimation

Pulp/tooth area

Incisors

Portugal 


\section{a b s t r a c t}

Since 2004, several papers on the analysis of the apposition of secondary dentine have been published. The aim of this paper was to study a sample of peri-apical X-ray images of upper and lower incisors, both lateral and medial, to examine the application of pulp/tooth area ratio as an indicator of age. A sample of 116 individuals, 62 men and 54 women, aged between 18 and 74 years, was studied. Data were fitted with age as a linear function of the pulp/tooth ratio of incisors. The total variance explained by the regression equation ranged from $51.3 \%$ of age, when lower lateral incisors were used as explanatory variable, to $81.6 \%$ when upper lateral incisors were used. The accuracy of the corresponding regression model yielded ME 1/4 8.44 and 5.34 years, respectively. These results show that, although incisors are less reliable than canines or lower premolars, they can be used to estimate age-at-death when the latter are absent. 


\section{Introduction}

The forensic literature has provided several methods for estimating age in adults, both dead and living. ${ }^{1,2}$ Many of these methods are macroscopic, ${ }^{3 e 5}$ only some are based on the study of teeth $^{6 \mathrm{e} 10}$ and a few on radiographic evaluation of both skeletal and dental districts. ${ }^{11-14}$ According to a recent study on current practices used by forensic anthropologists in adult skeletal age estimation, the Sucheye Brooks pubic symphysis method remains the preferred technique, cranial sutures and dental attrition or abrasion being the least frequently chosen, regardless of experience. ${ }^{15}$ Moreover, there is no standardized way of combining information from multiple age estimation methods into a final age range to present to the authorities requesting them. ${ }^{15}$ As several authors have noted, although historically age estimation has been regarded as "ultimately an art, not a precise science” (Maples, 1989), in the face of the Daubert challenge $\mathrm{e}^{16-18}$ and our current era of validation and scientific rigor, these problems need to be addressed. ${ }^{15}$

Thanks to recent and substantial advances in physical anthropology, old methods have been improved and new techniques have been proposed. ${ }^{2}$ Of these, analysis of the apposition of secondary dentine is of particular interest. ${ }^{19}$ After tooth eruption, it is wellknown that the size of the pulp cavity decreases gradually with age, because of the deposition of secondary dentine in the pulp cavity wall. ${ }^{20}$ This process is caused by the continual secretion of dentinal matrix by odontoblasts (physiological secondary dentinogenesis). Dentine is a living tissue containing odontoblasts which form the tooth. During a person’s lifetime, for both physiological and pathological reasons (attrition, abrasion, erosion, caries), the odontoblasts deposit layers of secondary dentine, which gradually obliterates the pulp chamber. The mean rates of increasing dentinal thickness have been found to be 6.5 $\mathrm{mm} /$ year for the crown and $10 \mathrm{~mm} /$ year for the root. The effect is a progressive increase in 
dentinal thickness of $0.45 \mathrm{~mm}(17.1 \%)$ and $0.60 \mathrm{~mm}$ (24.3\%) in the crown and root areas, respectively.20 However, as Murray et al. ${ }^{20}$ noted, age-related differences are observed between different tooth types: the increase in dentinal thickness in the crown aspect of canine teeth may be as low as p 3.4\%, whereas that of the crown of incisors and premolars may be $15.5 \%$ and $34.1 \%$ respectively, in older patients. Irrespective of age, mean dentinal thickness is observed to vary significantly across tooth types. Since 1925, when Bodecker ${ }^{21}$ ascertained that the apposition of secondary dentine was correlated with chronological age, new detailed studies of the pattern and rate of secondary dentine apposition in upper and lower anterior teeth have been performed. Secondary dentine deposition was included in the method pioneered by Gustafson, ${ }^{22}$ in which dentine transparency and secondary dentine values showed the highest correlation with age. Philippas $^{23}$ was one of the first to use the radiographic method to verify the influence of age on the formation of dentine. In 1995, Kvaal et al. ${ }^{24}$ developed a new method for estimating age in adults, based on the relationship between age and pulp size on peri-apical dental radiographs. Paewinsky et al. ${ }^{25}$ also tested the method of Kvaal et al. ${ }^{24}$ on digital panoramic radiographs, but specific regression formulas were developed by these authors for their sample. Currently, thanks to some conventional techniques, such as standard radiographs, or newly developed ones, such as micro-focus X-ray computed tomography, apposition of secondary dentine is a useful tool for age estimation in adults. ${ }^{2}$ Among these techniques, dental radiography is a convenient, simple, and much less expensive method which a legal medical expert can apply practically in several types of situations. A destructive approach, while more accurate, may not be acceptable in many forensic cases, due to the loss of evidence, or in living subjects in whom the tooth must be sacrificed. ${ }^{2}$ 
Since 2004, Cameriere et al. ${ }^{26,27}$ have published several papers on a method of age estimation using the pulp/tooth area ratio to quantify the apposition of secondary dentine. The canine was the first tooth studied by Cameriere et al. ${ }^{26}$ and Cameriere and Ferrante. ${ }^{26}$ Canines were chosen for a number of reasons: they are often present in old age, they are less likely than other anterior teeth to suffer attrition or abrasion as a result of particular work, and they are the single-root teeth with the largest pulp area and thus the easiest to analyse. ${ }^{26}$ Although this tooth type proved to be very reliable in earlier publications,28 lower premolars were later considered. ${ }^{29}$ These single-rooted teeth have a pulp size which is more evident than in other teeth, and they are comparable to canines. They are less easily damaged by direct heat or traumatic force than incisors or canines, as they are covered by the soft tissue of the cheek, and are not as easily lost in dry skull material as single-rooted anterior teeth. ${ }^{29}$ They show good correlations between the decrease in dental pulp and increasing age. $^{29}$

After analysis of canines and premolars, this study focused on the remaining group of single-rooted teeth, i.e., the upper and lower central and lateral incisors. As a rule, anterior teeth tend to be extracted before canines, but for aesthetic reasons patients wish to keep their front teeth as long as possible. The anterior teeth are much smaller and have less pulp than canines, particularly the lower incisors. In addition, variations in the root canal system of the lower incisors are quite high: over $40 \%$ of these teeth have two canals. ${ }^{30,31}$ The smaller pulp and the presence of a second canal, which cannot be observed in frontal periapical X-ray, reduce the probability of correct measurements and, consequently, accuracy in age estimation. This reduced pulp and the great variation in the presence or absence of a second and/or later canal had already been noted in previous studies, ${ }^{30-35}$ thus directing research toward study of canines and premolars. 
The aim of this paper is to study a sample of peri-apical X-ray images of upper and lower incisors, both lateral and central, to examine the application of the pulp/tooth area ratio as an indicator of age. This information would be very helpful as a reference for clinical root canal therapy and for age identification of skeletal remains in forensic human identification. $^{1,2}$

\section{Materials and methods}

\subsection{Samples}

Samples come from two of the Coimbra Identified Osteological Collections in the Department of Life Sciences at the University of Coimbra (Portugal): the Medical School Skull Collection and the International Exchange skull collections.

The skulls in the Medical School collection were acquired from the Schools of Medicine in Lisbon and Porto and from the Anatomical Theater of the University of Coimbra (Portugal). It is composed of 585 complete skulls, collected between 1895 and 1903 by Bernardino Machado, and was the first identified osteological collection to be amassed in Coimbra. $^{36}$

The skulls from the International Exchange collection were recovered from the Cemitério Municipal da Conchada in Coimbra (Portugal). This collection was probably initiated in 1915 by E. Tamagnini, who actively participated in accessions until $1942 .^{36}$ In Portuguese cemeteries, it is common practice to perform exhumations after a period of five years, and bones are transferred for deposition in an ossuary. However, if relatives do not claim the remains after exhumation or if they cease payment of ossuary fees, the remains are either placed in a communal grave or cremated. In these cases, the University of Coimbra may intervene and request the remains for research purposes. ${ }^{36}$ 
The individuals in the above-mentioned collections died between the years 1895e1903 and 1904e1938, respectively. As they were fully identified, detailed information about each of them - date and place of birth, sex, age at death, year and place of death, illness or cause of death, and occupation, and any other pertinent details e were compiled in record books. These records clearly indicate the low socio-economic status of most of the individuals: the women were almost all housewives, and the men were mainly employed as farm workers and artisans. The provenance of their bodies may also be considered a sign of their low socio-economic status, because in most cases their families could not afford adequate burial. ${ }^{36}$ From these collections, random samples of 116 individuals were made, 62 men and 54 women, aged between 18 and 74 years. In total, peri-apical X-ray of 427 lateral and central incisors were taken: 19\% are central upper, 26\% lateral upper, 28\% central lower and $27 \%$ lateral lower. Of all incisors, $43.3 \%$ belonged to women and $56.7 \%$ to men. The inclusion criteria were: periapical X-ray selected from subjects aged between 18 and 75 years; the selected tooth on the radiograph, the maxillary incisor, was fully erupted into the oral cavity; the root of the incisor was fully formed. The exclusion criteria were: teeth showing signs of root canal treatment, impacted teeth, and teeth with vestibular radioopaque fillings, crowns or pathological processes visible on the peri-apical radiograph. Radiographs showing badly rotated teeth or teeth with large areas of enamel overlap between neighboring teeth were also excluded. The study samples also show minimal attrition. The distributions of tooth type according to subjects' sex and age at death are listed in Table 1.

The selected nomenclature to classify the teeth is that proposed by the Fédération Dentaire Internationale (FDI). Protocols to collect radiographs for human subjects were approved by the Ethics Committee for Research Involving Human Subjects of the University of 
Coimbra, and the study was conducted in accordance with the ethical standards laid down by the Declaration of Helsinki (Finland). The World Medical Association (WMA) developed the Declaration of Helsinki as a statement of ethical principles for medical research involving human subjects, including research on identifiable human material and data.

Peri-apical digital X-ray were taken on a NOMAD hand-held dental X-ray device (Aribex, USA) combined with a digital sensor (DSX, Anthos, Italy) linked to a portable computer. All radiographs were taken with a Rinn-type digital sensor holder, with $0.05 \mathrm{~s}$ exposure time at $60 \mathrm{kV}$. Following Cameriere et al., ${ }^{26}$ the X-ray images were photo-edited by Adobe Photoshop.

\subsection{Measurements}

Following Cameriere et al., ${ }^{26}$ each radiographic image was saved as a high-resolution JPEG file on a desktop computer and imported to the Adobe Photoshop CS4_image-editing software program (Adobe Systems Incorporated, San Jose, CA, USA). Next, the image file was opened; the working area enlarged, and zoomed in. Brightness/contrast and sharpness, if needed, were adjusted.

The polygonal lasso tool was selected from the tool bar. In order to select the entire incisor area, it was necessary to click on the incisor image in order to set the starting point of the incisor shape; then the cursor was moved to a close point of the tooth profile and clicked again. A straight line from the first point selected was drawn. Clicking continued to set endpoints for subsequent segments along the incisor profile. As reported by Paewinsky et al., ${ }^{25}$ a minimum of 20 points from each tooth outline was identified and connected with the line tool. The selected area was copied and pasted on a new layer, which was added to the 
active working area superimposed on the incisor image. This new layer, renamed "INCISOR", was added to the layer palette. In order to select the pulp chamber area, it was necessary to proceed as previously for the entire incisor, following the pulp chamber profile with the polygonal lasso. A minimum of 10 points was also marked on the pulp outline, although up to 15 points were marked in some cases. The pulp chamber selection was copied, pasted to a new layer, and renamed "PULP CHAMBER". The new "PULP CHAMBER" layer contained only the incisor pulp chamber area pixels, as the "INCISOR" layer contained the pixels for the entire incisor area. To know how many pixels there were in each layer, the histogram palette (windows > histogram) was activated and the "PULP CHAMBER" layer was selected by double-clicking on the layer name in the layer palette. In the histogram palette from the source menu, the SELECTED LAYER was chosen and a double-click on the histogram image was made. The number of pixels was shown in the "PULP CHAMBER" layer in the histogram palette. This value represented the first required variable (pulp chamber area). Next to be selected was the "INCISOR" layer, and a double-click was made on the histogram image. The number of pixels contained in the entire incisor represented the second required variable. ${ }^{26}$

\subsection{Intra- and inter-observer agreement}

Each file was numbered consecutively from 1 to 427, being part of a blind set-up. When analysing the radiographs, the observer did not know the chronological age of the individuals examined. All measurements were carried out by the same observer (RC) with ample experience of this technique. Since being able to replicate measurements reliably is an essential component of any metric study, tests for intra-observer error were performed. To evaluate intra-observer reproducibility, a random sample of 50 peri-apical radiographs 
was re-examined after an interval of at least 2 weeks and studied with the concordance correlation coefficient.

Lastly, in order to evaluate inter-observer error, a random subsample of 50 individuals was selected and re-examined after a period of 3 weeks by the first author (RC). This sample was also measured by a second observer, a forensic anthropologist with many years of experience in the fields of radiology, dental anatomy and forensic research, who had not worked with this method before. The sub-sample was composed of samples from 25 men and 25 women and the same procedure for all measurements was followed. ${ }^{26}$ Upon completion of data collection, all measurements were entered into a computer database. A paired-sample t-test was adopted ${ }^{37}$ to assess the rates of agreement between the two observers.

\subsection{Statistical analysis}

$\mathrm{RA}_{i j}(\mathrm{RA}=$ Ratio) was used to indicate the pulp/tooth area ratio of the tooth in position $i j$ ( $i$ $=1,2,3,4 ; j=1,2, .5$ ) in the FDI notation. For each radiograph, dental maturity was evaluated by measuring the pulp/tooth area ratio of incisors. Morphological variables $\mathrm{RA}_{1 \text { sup }}, \mathrm{RA}_{2 \text { sup }}, \mathrm{RA}_{1 \mathrm{inf}}$ and $\mathrm{RA}_{2 \text { inf }}$ were then used to indicate the pulp/tooth area ratio of the central and lateral upper and lower incisors. Data from left and right incisors were pooled, since previous works ${ }^{26}$ had shown no differences in the rate of development in contralateral teeth. $\mathrm{RA}_{1 \text { sup }}, \mathrm{RA}_{2 \text { sup }}, \mathrm{RA}_{1 \mathrm{inf}}$ and $\mathrm{RA}_{2 \text { inf }}$ and the individual's sex were entered in an EXCEL file, for use as predictive variables for dental age estimation in subsequent statistical analysis. Analysis of covariance (ANCOVA) was then applied to study interactions between age, incisors and sex. 
To evaluate the accuracy of a regression model, the known age of each subject $\left(\mathrm{Age}_{i}, i=\right.$ $1, .$, n) was compared with estimated age (Age est $, i, i=1, ., n)$ by the mean prediction error (ME) method, which represents the mean of the absolute values of the differences between chronological and estimated ages (residuals):

$$
M E=\frac{1}{\mathrm{n}} \sum_{\mathrm{i}-1}^{\mathrm{n}} E_{i}=\frac{1}{\mathrm{n}} \sum_{\mathrm{i}-1}^{\mathrm{n}}\left|\mathrm{Age}_{\mathrm{i}}-\mathrm{Age}_{\mathrm{est}, \mathrm{i}}\right|
$$

where $n$ is the number of subjects in the sample, and $\mathrm{E}_{i},(i=1, ., n)$ is the absolute value of the i-th residual, i.e., the difference between the CA (chronological age) and DA (dental age) of the i-th individual:

$$
\delta_{\mathrm{i}}=\text { Age }_{i}-\text { Age }_{\text {est }, i}, i=1, \ldots, n .
$$

A positive value (+) of $\delta_{\mathrm{i}}$ indicates underestimation and a negative value (-) overestimation. All statistical analyses were performed with the R statistical program (version 2.14.0) ${ }^{37}$ and Microsoft Excel ${ }^{\circledR}$. Significance level was set at 5\%.

\section{Results}

Intra-observer agreement was over $95 \%$ of cases. There were no significant inter-observer differences $(p=0.231)$.

For measurements of the pulp/tooth area ratio of incisors, the ANCOVA (Table 2) revealed a significant difference in the intercept between men and women $(p<0.05)$. The data were consequently fitted according to the following linear model:

$$
\text { Age }=\beta_{0}+\beta_{1} \cdot g+\beta_{2} \cdot \text { RA }
$$


where binary variable g represents an individual's sex, and is assigned a value of 1 if the individual is male and 0 otherwise, and RA represents the pulp/tooth ratio of the incisors. The regression lines for men and women and for each incisor type are shown in Fig. 1. The parameter estimates of the linear model with equal slope but different intercepts for male and female individuals are listed in Table 3, together with their standard errors. The resulting regression equations, with single type of incisors, are listed in Table 4.

When lower lateral incisors were used as explanatory variables for age estimation, the regression equation explained only $51.3 \%$ of the total variance of age $\left(R^{2}=0.513\right)$, with a standard estimate error of 10.9 years. However, as shown in Table 4, when upper lateral incisors were used, the percentage of the explained variance rose to $81.6 \%$ and the standard estimate error fell to 6.64 years. When the predictive variable was $\mathrm{RA}_{1 \text { sup }}, \mathrm{RA}_{2 \text { sup }}, \mathrm{RA}_{1 \mathrm{inf}}$ or $\mathrm{RA}_{2 \mathrm{inf}}$, the accuracy of the corresponding regression model gave $\mathrm{ME}=5.81,5.34,8.01$ and 8.44 years, respectively.

The regression lines (Fig. 1) show that the regression models fit the data trend reasonably well. The residual plots (Fig. 2) do not show any obvious pattern, although the interquartile range of residuals varies from 13.19 years, when the explained variable refers to the lower lateral incisor, to 9.44, for the upper lateral incisor.

\section{Discussion}

Assessment of the pulp/tooth area ratio is an indirect quantification of secondary dentine

deposition. ${ }^{2,26}$ The ratio between tooth and pulp measurements was calculated and used in order to reduce the effect of any variation in the magnification or angulations of the radiographs. ${ }^{26,27}$ 
In this study, upper and lower incisors were analysed to establish the patterns of the relationship between age and the apposition of secondary dentine in a contemporary Portuguese sample. Unlike canines and premolars, ANCOVA analysis applied to possible interactions between age, incisors and sex, showed that sex affects intercepts in linear regression models (see Equation (1)). In particular, the results also show different kinds of aging: straight lines for men and women, and a uniformly slower rate in women (Fig. 1). Previous studies ${ }^{2,26,27}$ on other single-root teeth have shown that sex has no significant influence on age estimation, whereas the pulp/tooth ratios of female incisors are significantly smaller than those of men. In this study, the difference between men and women ranges from 0.009 (upper lateral) to 0.018 (lower lateral), which corresponds to a drop ranging from 2.99 to 6.11 years. In their study on dental age estimation by calculation of pulp/tooth volume ratios of single-rooted teeth, Star et al. ${ }^{38}$ demonstrated that the observed relation between the pulp/tooth volume ratio and age was stronger for women than for men, but that the difference in relation was not significant $(\mathrm{p}=0.86)$; nor did they observe any significant interaction between tooth types and sex $(p=0.50)$. However, in the study of Zaher et al., ${ }^{39}$ on age estimation from the pulp/tooth area ratio in maxillary incisors, ANCOVA analysis showed that sex did not contribute significantly to the pulp/tooth ratio $(\mathrm{F}=0.79, \mathrm{p}=0.74$ for central incisors, and $\mathrm{F}=0.74, \mathrm{p}=0.77$ for lateral incisors). Someda et al. ${ }^{33}$ analysed accuracy by sex, finding higher accuracy for women compared with men, and also observed sex differences for $95 \%$ confidence intervals in the estimation of population regressions for each volume ratio. Kvaal et al. ${ }^{24}$ performed multiple linear regression analysis for age and the width and height ratios of the pulp cavity, and observed statistically significant sex differences for lower lateral incisors. 
This dependence of estimated age on sex adds a further difficulty, because it means that the sex of the subject whose age is to be estimated must be known. This knowledge is more easily obtained if the age-at-death of a complete skeleton is available, but may be more difficult if the remains are not well preserved and/or the skeleton is incomplete. ${ }^{2,40}$ Regarding accuracy, the regression model yielded ME = 8.44 and 5.34 years, respectively, when lower and upper lateral incisors were used as explanatory variables. As shown in the study of Du et al. ${ }^{41}$ on age-related changes in the pulp cavity of incisors, narrowing velocity is unequal according to tooth position. In the maxillary incisors, the decrease in the mesiodistal diameters of the cervical pulp chamber and the middle part of the root canal is about $0.02 \mathrm{~mm}$ per year $(b=-0.02 \mathrm{~mm})$, twice as fast as in the lower incisors. This is consistent with the results of Schroeder et al., ${ }^{35}$ who studied the age-related restriction of the pulp chamber and the structure of the inorganic dentinal wall of the pulp chamber in upper and lower incisors by X-ray and scanning electron microscopy. Their results demonstrated that, with increasing age, the pulp chamber, in particular the root canal, becomes restricted in the mesio-distal direction but not, or only much later, in the vestibule oral direction. This is because a network of tubule-free fibrodentine or more regular secondary dentine, with or without tubules, is deposited on these pulp chamber walls. In the vestibular and oral aspects of the dentinal walls, the density of the tubules in the upper incisors is higher than in the lower incisors at the mesial and distal walls. Tubule density also decreases, mainly from the middle of the root canal towards the apex. This suggests that narrowing of the pulp cavity in the upper incisors may serve as a more sensitive indicator of age estimation. During this study, it was evident that the analysis of the pulp/root area ratio for age estimation has certain limitations. It cannot be used in multi-rooted teeth, as accurate measurements of such teeth are difficult to perform. Similarly, as the curved arch of the jaw 
is projected on a flat film, there is always a certain amount of distortion when images are presented. It was to compensate for this that periapical radiographs are now used. More accurate radiographic images can be produced by both paralleling and bisecting angle techniques. The paralleling technique produces low image distortion. The roots and crowns of canines can be clearly shown, enabling study of teeth and pulp chambers. The resulting digitalized images show greater contrast and the possibility of overlap is reduced. In addition, instead of absolute measurements, in order to minimize differences in magnification of radiographs and angulations between X-ray beams and film, the ratio between pulp area and tooth can be analysed.

As regards the meaningfulness and usefulness of these results in the legal sphere, this research shows promising results for noninvasive dental age estimation by the use of dental radiographs from upper and lower incisor teeth, although a more population specific equation must be generated in order to improve its accuracy. In addition, in view of the above results, it is very important to standardize methods for assessment of secondary dentine. Further studies with larger sample groups are therefore recommended, in order to verify not only the accuracy of the method but also inter-observer agreement. The radiographic technique must be clearly defined, so that it can be applied by forensic researchers all over the world. ${ }^{2}$ In unidentified deceased individuals, this method would be useful in conjunction with age indicators in other areas of the body. When living adult individuals are evaluated for age, analysis of the apposition of secondary dentine is a non-invasive, quick and economic biometric technique.

\section{Conclusions}


The present study investigated peri-apical X-ray measurements of both upper and lower incisors, to examine the relationship between age and age-related changes in the pulp/tooth area ratio.

The results demonstrated that the variability in age explained by the pulp/tooth area ratio in incisors is affected by sex. In addition, when age estimation was performed according to age-related changes in the pulp/tooth area ratio, more accurate estimations were obtained by analysing upper lateral incisors. This is because the narrowing velocity in the pulp chamber of these teeth is twice as fast as that in the lower incisors.

According to this finding, it may be stated that incisors are less reliable than canines or lower premolars. However, the pulp/tooth area ratio in incisors is an age-dependent variable which can be used to estimate age with reasonable accuracy, especially in combination with other age indicators or when other single-rooted teeth are absent.

\section{Conflict of interest}

Neither the author nor any of the coauthors have any potential conflict of interests related to the publication of this paper.

\section{References}

1. Baccino E, Schmitt A. Determination of adult age at death in the forensic context. In: Schmitt A, Cunha E, Pinheiro J, editors. Forensic anthropology and medicine. New York: Humana Press; 2006. p. 259-80.

2. Cunha E, Baccino E, Martrille L, Ramsthaler F, Prieto JL, Schuliar Y, et al. The problem of ageing human remains and living individuals: a review. Forensic Sci Int 2009;193:1-13. 
3. Baccino E, Ubelaker DH, Hayek LA, Zerilli A. Evaluation of seven methods of estimating age at death from mature human skeletal remains. J Forensic Sci 1999;44(5):931-6.

4. Ritz-Timme S, Cattaneo C, Collins MJ, Waite ER, Schutz HW, Kaatsch HJ, et al. Age estimation: the state of the art in relation to the specific demands of forensic practise. Int $\mathrm{J}$ Legal Med 2000;113(3):129-36.

5. Schmeling A, Grundman C, Fuhrman A, Kaatsch HJ, Knell B, Ramstahler F, et al. Criteria for age estimation in living individuals. Int J Legal Med 2008;122(6): 457-60. 6. Lamendin H, Baccino E, Humpert JF, Tavernier JC, Nossintchouk RM, Zerilli A. A simple technique for age estimation in adult corpses: the two criteria dental method. J Forensic Sci 1993;37(5):1373-9.

7. Yekkala R, Meers C, Van Schpdael A, Hoogmartens J, Lambrichts I, Willems G. Racemization of aspartic acid from human dentin in estimation of chronological age. Forensic Sci Int 2006;159(Suppl. 1):S89-94.

8. Baccino E, Zerilli A. The two step strategy (TSS) or the right way to combine a dental (Lamendin) and an anthropological (Suchey-Brooks system) method for age determination (abstract). In: Proceedings of the 49th annual meeting of the American Academy of Forensic Sciences. New York, NY: Colorado Springs: CO American Academy of Forensic Sciences; 1997.

9. Ubelaker DH, Parra R. Dental aging methods and population variation as demonstrated in a Peruvian sample. In: Proceedings American Academy of Forensic Sciences, vol. XIV. Washington DC; 2008. p. 341.

10. Spalding KL, Buchholz BA, Bergman LE, Druid H, Frisèn J. Forensics: age written in teeth by nuclear tests. Nature 2005;437:333-4. 
11. Schulz R, Muhler M, Mutze S, Schmidt S, Reisinger W, Schmeling A. Studies on the time frame for ossification of the medial epiphysis of the clavicle as revealed by CT scans. Int J Legal Med 2005;119:142-5.

12. McCormick WF, Stewart JH. Age related changes in the human plastron: a roentgenographic and morphologic study. J Forensic Sci 1988;33:100-20.

13. Landa MI, Garamendi PM, Botella MC, Alemán I. Application of the method of Kvaal, et al. to digital orthopantomograms. Int J Legal Med 2009;123(2):123-8.

14. Bosmans N, Ann P, Aly M, Willems G. The application of Kvaal’s dental age calculation technique on panoramic radiographs. Forensic Sci Int 2005;153: 208-12. 15. Garvin HM, Passalacqua NV. Current practices by forensic anthropologists in adult skeletal age estimation. J Forensic Sci 2012;57(2):427-33.

16. Daubert V. Merrell Dow Pharmaceuticals; 1993. Inc, 509 US 579.

17. Christensen AM. The impact of Daubert: implications for testimony and research in forensic anthropology (and the use of frontal sinuses in personal identification). J Forensic Sci 2004;49(3):427-30.

18. Gatowski S, Dobbin SA, Richardson JT, Ginsburg GP, Merlino ML, Dahir V. Asking the gatekeepers: a national survey of judges on judging expert evidence in a post-Daubert world. Law Hum Behav 2001;25(5):433-58.

19. Solheim T. Amount of secondary dentine as an indicator of age. Scand J Dent Res 1992;100:193-9.

20. Murray PE, Stanley HR, Matthews JB, Sloan AJ, Smith AJ. Age-related odontometric changes of human teeth. Oral Surg Oral Med Oral Pathol Oral Radiol Endod 2002;93(4):474-82. 
21. Bodecker CF. A consideration of some of the changes in the teeth from young to old age. Dental Cosmos 1925;67:543-9.

22. Gustafson G. Age determinations on teeth. J Am Dent Assoc 1950;41:45-54.

23. Philippas GG. Influence of occlusal wear and age on formation of dentin and size of pulp chamber. J Dent Res 1961;40:1186-98.

24. Kvaal SI, Kolltveit KM, Thomsen IO, Solheim T. Age estimation of adults from dental radiographs. Forensic Sci Int 1995;74:175-85.

25. Paewinsky E, Pfeiffer H, Brinkmann B. Quantification of secondary dentin formation from orthopantomograms. A contribution to forensic age estimation methods in adults. Int $\mathrm{J}$ Legal Med 2005;119:27-30.

26. Cameriere R. AgEstimation project: Cameriere’s methods for age estimation. Macerata (Italia): Eum, Edizioni Università di Macerata; 2008.

27. Cameriere R, Cunha E, Sassaroli E, Nuzzolese E, Ferrante L. Age estimation by pulp/tooth area ratio in canines: study of a Portuguese sample to test Cameriere's method. Forensic Sci Int 2009;193(1e3):128.e1-6.

28. De Luca S, Bautista J, Alemán I, Cameriere R. Age-at-death estimation by pulp/tooth area ratio in canines: study of a 20th-century Mexican sample of prisoners to test Cameriere’s method. J Forensic Sci 2011;56(5): 1302-9.

29. Cameriere R, De Luca S, Alemán I, Ferrante L, Cingolani M. Age estimation by pulp/tooth ratio in lower premolars by orthopantomography. Forensic Sci Int 2011;214:105-12.

30. AI-Qudah AA, Awawdeh LA. Root canal morphology of mandibular incisors in a Jordanian population. Int Endod J 2006;39:873-7. 
31. Sert S, Aslanalp V, Tanalp J. Investigation of the root canal configurations of mandibular permanent teeth in the Turkish population. Int Endod J 2004;37: 494-9.

32. Pecora JD, Savioli RN, Murgel CAF. Study of the incidence of two root canals in human mandibular incisors. Rev Bras Odont 1990;47:44-7.

33. Someda H, Saka H, Matsunaga S, Ide Y, Nakahara K, Hirata S, et al. Age estimation based on three-dimensional measurement of mandibular central incisors in Japanese.

Forensic Sci Int 2009;185(1e3):110-4.

34. Hwang YH, Min KS. Clinical management of 3 mandibular incisors with 2 separate canals and foramina: case report. J Can Dent Assoc 2005;71: 388-9.

35. Schroeder HE, Krey G, Preisig E. Age-related changes of the pulpal dentin wall in human front teeth. Schweiz Monatsschr Zahnmed 1990;100(12): 1450-61.

36. Cunha E, Wasterlain SN. The Coimbra identified osteological collections. In: Grupe G, Peters J, editors. Skeletal series and their socio-economic context. Documenta Archaeobiologiae, Vol. 5. Rahden/Westf: Verlag Marie Leidorf GmbH; 2007. p. 23-33. 37. R Development Core Team. R: a language and environment for statistical computing. Vienna, Austria: R Foundation for Statistical Computing, ISBN 3-900051-07-0; 1998. URL, http://www.r-project.org; 1998 [last accessed 07.02.12].

38. Star H, Thevissen P, Jacobs R, Fieuws S, Solheim T, Willems G. Human dental age estimation by calculation of pulpetooth volume ratios yielded on clinically acquired cone beam computed tomography images of monoradicular teeth. J Forensic Sci 2011;56:77-82. 39. Zaher JF, Fawzy IA, Habib SR, Ali MM. Age estimation from pulp/tooth area ratio in maxillary incisors among Egyptians using dental radiographic images. J For Legal Med 2011;18:62-5. 
40. Maples WR. The practical application of age-estimation techniques. In: Is, can MY, editor. Age markers in the human skeleton. Springfield IL: Charles C. Thomas; 1989. p. 319-24.

41. Du C, Zhu Y, Hong L. Age-related changes in pulp cavity of incisors as a determinant for forensic age identification. J Forensic Sci 2011;56:72-6. 